\title{
TAPIDO: Trust and Authorization Via Provenance and Integrity in Distributed Objects (Extended Abstract) ${ }^{\star}$
}

\author{
Andrew Cirillo, Radha Jagadeesan, Corin Pitcher, and James Riely \\ School of CTI, DePaul University
}

\begin{abstract}
Existing web services and mashups exemplify the need for flexible construction of distributed applications. How to do so securely remains a topic of current research. We present TAPIDO, a programming model to address Trust and Authorization concerns via Provenance and Integrity in systems of Distributed Objects. Creation of TAPIDO objects requires (static) authorization checks and their communication provides fine-grain control of their embedded authorization effects. TAPIDO programs constrain such delegation of rights by using provenance information. A type-and-effect system with effect polymorphism provides static support for the programmer to reason about security policies. We illustrate the programming model and static analysis with example programs and policies.
\end{abstract}

\section{Introduction}

Web services, portlets, and mashups are collaborative distributed systems built by assembling components from multiple independent web applications. Building such systems requires programming abstractions that directly address service composition and content aggregation. From a security standpoint, such composition and aggregation involves subtle combinations of authentication, authorization, delegation, and trust.

The issues are illustrated by account aggregation services that provide centralized control of an individual's accounts held with one or more institutions. An individual first grants permission for an aggregator to access owned accounts located at various institutions. In a typical use case, the aggregator is asked to provide a summary balance of all registered accounts: the aggregator asks each institution for the relevant account balance; the institution then determines whether or not to grant access; with the accumulated balances, the aggregator returns a summary of registered accounts to the individual. This simple service already raises several security and privacy issues related to trust and authorization. To name just two:

- The account owner's intent to access their account should be established by the institution. Message integrity is required to verify such intent.

- Principals should establish that the flow of messages through the system complies with authorization, audit, and privacy policies for account access. Message provenance is required to verify that the message history does comply with such policies.

\footnotetext{
* Companion technical report available at http://www.teasp.org/tapido/. Andrew Cirillo and James Riely were supported by NSF Career 0347542. Radha Jagadeesan and Corin Pitcher were supported by NSF Cybertrust 0430175.
} 


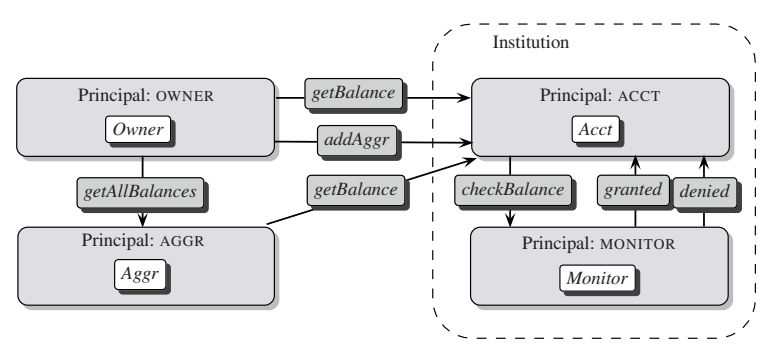

Fig. 1. Principals Involved in Account Aggregation

It has been said that "An application can be mashup-friendly or it can be secure, but it cannot be both." [1]. We disagree. In this paper, we describe the use of message provenance and integrity to achieve both security and flexibility aims in this general programming context. In this extended abstract, we focus on the expressive power of the programming model using examples. For a complete formal treatment of the static analysis, please see the companion technical report.

In the remainder of this section, we present an informal overview of our approach using the account aggregation example. The principals involved are the account owner, the aggregation service, and two principals for the institution holding the account. The institution uses two principals to distinguish privileged monitor code from public-facing, unprivileged code. The owner requests the balance from the public-facing account object, which in turn contacts a trusted monitor to determine whether access should be granted or denied. The flow of messages is summarized in Figure 1

Object model. TAPIDO's object model is based upon Java's notion of remote objects. We locate objects at atomic principals. Examples of atomic principals are nodes on a distributed system, a user or a process. For an object $p$, the location is available to the programmer via $p$.loc. As with Java's remote objects, objects are immobile and rooted at the location where they are created. A method invocation on an object leads to code execution at the location of the callee object. Thus, when the caller and callee objects are located at different locations, method invocation leads to a change of location context. References to objects are mobile - they can be freely copied and they move around through the system as arguments to methods or return values. We do not address mobility of objects themselves; thus, we do not discuss serialization and code mobility.

TAPIDO assumes a communication model that guarantees the provenance and integrity of messages. Thus, TAPIDO focuses on semantic attacks on trust and authorization, rather than on attacks against the cryptographic techniques required to achieve this communication model. Thus, our approach assume an underlying network model in which the sender of the message can be reliably determined; this model is wellstudied [2 3 4 45] and realizable [678]. Using a relatively high-level model permits us to concentrate on attacks that seek unauthorized access, rather than studying the underlying cryptographic protocols that facilitate the integrity assumption.

Statics. Effects are communicated through object references. The language of effects is a decidable monotonic fragment of first-order logic (e.g., Datalog) extended to work 
over authorization logics. The modalities of authorization logics [9 10 11]12] permit different participants of a distributed system to maintain potentially inconsistent worldviews, e.g. if $b$ receives an object with effect $\phi$ created by $a$, it receives the effect $a$ says $\phi$, rather than the more absolute truth $\phi$. Our language of effects also includes logic variables to achieve ML-style polymorphism with respect to effects.

Our "object-centric" notion of effects differs from the more usual "method-centric" notions explored in the literature on effects in Object-Oriented (OO) languages. The effects on objects can only refer to the immutable data of the object - if the object is an authorization token, this effect can record the rights associated with these object. For honest agents, object effects are validated at the point of creation, effectively ensuring that the global policy permits the creation of the object. When such an object is received - e.g., as an argument to a method call - the effects are transferred as a benefit to the recipient. In any execution of a well-typed program, there is a corresponding [13] object creation validating such accrual of rights.

The attackers that we consider are untrustworthy atomic principals running any welltyped Java program. Following [14] and our own earlier work [15], they may "utter" anything whatsoever in terms of effects. For example, opponents may create authorization objects without actually having the rights to create them, aiming to subvert the global authorization policy. A program is safe [16] if every object creation at runtime is justified by the accumulated effects. Our type system ensures that well-typed programs remain safe under evaluation in the face of arbitrary opponent processes.

In the account aggregation example, consider when an individual requests their balance from the institution holding their account through the aggregator. The guarantee sought is that the institution may only respond with the account balance when the request is approved by the account owner. With a pre-arranged protocol, approval can be conveyed by a message passed from the account owner to the institution via the aggregator. The institution's code must be able to verify that it originates with the owner and not been modified en route. The code must also ensure that the integrity-verified message and the pre-arranged protocol entail the owner's approval in the past; even in the presence of attackers who (perhaps falsely) claim possession of rights.

We describe a program incorporating such a design in our model, and verify the required properties with our static analysis.

Programming Provenance. Provenance - the history of ownership of an object has received much interest in databases, e.g., see [17] for a survey. Security-passing style implementations [18] of stack inspection are already reminiscent of such ideas in a security context, since the provenance of the extra security-token parameter can be viewed as encoding the current relevant security context.

Provenance plays a crucial role in both the privacy architecture and the security (access control and accountability) of the account aggregation example. Consider the request from the account owner to the institution via the aggregator. The institution may impose an access control policy on the provenance of the request, e.g., to restrict the aggregators that can be used with the institution's services. Such a policy is distinct from, but can be used in conjunction with, an access control policy based upon the originator of the request. Similarly, the institution's audit policy may require a record of the provenance of requests (including the identities of the owner and the aggregator) to support 
an accountability obligation, e.g., to explain why and to whom account information was provided should the institution be accused of dishonest behavior.

Finally, the account owner can demand security of the path traversed by the result of such a request to ensure data privacy. This is demonstrated to the account owner by returning the relevant snapshot of the history of their data along with their data.

In contrast to stack inspection and history-based access control (e.g., see [19]) that mandate the flow of the security token, and record in it the full history of information used to make a judgement, our "user-defined" approach relies on trust relationships between the principals that are recorded as part of the history to make judgements.

In the account aggregation example, the response from the institution to the account owner has full history that can be described with the regular expression ACCT $\cdot$ trusted ${ }^{*}$. AGGR trusted $^{*} \cdot$ OWNER, where trusted represents a collection of trusted principals. Our explicit programming of this path in the sequel maintains only a subsequence of the history that matches ACCT $\cdot$ trusted ${ }^{*} \cdot$ AGGR $\cdot$ OWNER. Such abbreviations of the full history are codified in the security policy by assumptions on these principals - e.g., that the aggregator received the result from a trustworthy principal that can be relied upon to enforce the policy, and that the aggregator can be relied upon to report this information accurately.

We describe a program incorporating such a design in our model, and verify the required properties with our static analysis.

Related work. The study of effect systems was initiated in the context of functional languages (e.g., see Gifford and Lucassen [2021], and Talpin and Jouvelot [22|23] amongst others). The ideas have since been applied broadly to OO languages; to name but a few, specifying the read/write behavior of methods [24 25], confinement [2627], type reclassification [28], object protocols [29] and session types [30].

The most closely related papers are types for authorization, by Fournet, Gordon and Maffeis [31], a successor paper by the same authors [14] and our own earlier paper [15]. All of these papers (including this one) focus on authorization issues and so the work on information flow, e.g., see [32] for a survey, is not directly relevant. However, as in information flow based methods, TAPIDO global policy drives program design.

Fournet, Gordon and Maffeis [31] introduce an assume-guarantee reasoning framework with Datalog assertions for dealing with types for authorization. Both papers [3114 are based in a pi-calculus formalism and view authorization as "a complex cryptographic protocol" [31] in the context of the traditional "network is the opponent" model. The successor paper uses dependency analysis on authorization logic to formalize a subtle notion of security despite compromise. Our object-centric effects adapt their static annotations to an $\mathrm{OO}$ setting. Our requirements on object creation (resp. transfer of effects to the callee) are analogous to their expectation (resp. statement) annotations.

Our prior paper [15] was inspired by [31]. It was also placed in a mobile process calculus, but diverged from [3114] in assuming a model with explicit identities and a network that guaranteed integrity.

In this paper, we study imperative distributed objects by building on these intuitions. Our primary aim in this paper is to provide foundations of a programming methodology to ensure that distributed systems validate authorization and security policies; e.g., one of the aims of our examples is to illustrate the use of standard OO mechanisms 
to incrementally construct security guarantees. While the pi-calculus (with notions of keys) is expressive enough to code distributed objects (with explicit identities), such a translation is arguably inconsistent with our overall aims - just consider the complex encoding of state in the control of a pi-program. Such a translation based semantics approach obfuscates the simple (from an object standpoint) invariants that underlie our analysis. At any rate, the type systems in these three papers do not include the invariants of processes required to capture the type annotations of TAPIDO.

\section{Language}

We present the evaluation semantics for TAPIDO, a distributed class-based language with mutable objects. Our treatment of classes follows earlier direct semantics for classbased languages [33 34 [24 35]. We do not address issues of genericity [36|34] or inner classes [37]. Our treatment of concurrency follows Gordon and Hankin's concurrent object calculus [38]. As in Cardelli's Obliq [39], our object references have distributed scope, rather than local scope [40]. Our treatment of locations borrows heavily from process algebras with localities (see [41] for a survey).

We first describe our naming conventions. Names for classes $(c, d)$, methods $(\ell)$, fields $(f, g)$, variables $(x, y, z)$, objects $(p, q)$ and principals $(a, b)$ are drawn from separate namespaces, as usual. Predicate variables $(\alpha, \beta)$ and predicate constructors $(\gamma)$ occur in static annotations used during type-checking.

The reserved words of the language include: the variable names "this" and "caller"; the binary predicate constructors " $\wedge$ ", representing conjunction, and "says", representing quoting; the ternary predicate constructor Prov is used to indicate that the first argument (an object) was received from the second argument (source principal) by the third argument (target principal). We write the binary constructors infix.

The language is explicitly typed. Object types $(c<\vec{\phi}>)$ include the actual predicate parameters $\vec{\phi}$, which we treat formally as extended values. Value types include objects $(C)$, principals (Prin) and Unit. Extended value types include predicate types $(P)$, which are resolved during typechecking. The process type (Proc) has no values.

\begin{tabular}{ll}
\hline$C, D::=c<\vec{\phi}>$ & Object Types \\
$T, S::=C \mid$ Prin $\mid$ Unit & Value Types \\
$P, Q::=\operatorname{Pred}(\overrightarrow{\mathscr{T}})$ & Predicate Types \\
$\mathscr{T}, \mathscr{S}::=T|P|$ Proc & Types \\
$\mu::=$ final $\mid$ mutable & Mutability Annotations \\
$\mathscr{D}::=\operatorname{class} c<\vec{\alpha}: \vec{P}>\triangleleft D\{\vec{\mu} \vec{T} \vec{f} ; \overrightarrow{\mathscr{M}}\}[\theta]$ & Classes $(\vec{\alpha}$ bound in $D, \theta, \vec{T}, \overrightarrow{\mathscr{M}})$ \\
$\mathscr{M}::=\langle\vec{\beta}: \vec{Q}>S \ell(\vec{T} \vec{x})\{M\}$ & Methods $(\vec{\beta}$ bound in $S, \vec{T}, M ; \vec{x}$ in $M)$ \\
\hline
\end{tabular}

One may write classes and methods that are generic in the predicate variables, achieving ML-style polymorphism with respect to effects. Class declarations thus include the formal predicate parameters $\vec{\alpha}$, which may occur in the effect $\theta$ (see next table) associated with instances of the class. In addition to effects, class declarations include field 
and method declarations, but omit implicit constructor declarations. Fields include mutability annotations, which are used in the statics. The syntax of values and terms is as follow:1.

\begin{tabular}{cc}
\hline$V, W, U, A, B, \phi, \psi::=$ & Open Extended Values \\
$x|p| a \mid$ unit & Variable, Runtime Value \\
$\alpha|\gamma| \phi(\vec{V}) \mid \cdots$ & Predicates \\
$M, N, L, \theta::=$ & Terms \\
$V \mid$ new $c<\vec{\phi}>(\vec{V})$ & Value, Object Creation \\
let $x=V \cdot \ell<\vec{\phi}>(\vec{W}) ; M|V \cdot f| V \cdot$ loc $\mid V \cdot f:=W$ & Object Operations \\
if $V=W$ then $M$ else $N \mid$ let $x=N ; M \mid N \| M$ & Control Flow \\
$p: c\{\vec{f}=\vec{V}\}|(\nu p: C) M| a[M]$ & Runtime Terms \\
\hline
\end{tabular}

We use the metavariables $\phi, \psi$ and $\theta$ to represent values and terms of predicate type, and the other metavariables to represent runtime values and terms, with $A$ and $B$ reserved for values of principal type. Predicates are static annotations used in type-checking, which do not play any role in the dynamics.

An expectation "expect $\theta$ " may be written as "new Proof $\langle\theta>()$ ", where class Proof is defined "class Proof $<\alpha: \operatorname{Pred}>\{\}[\alpha]$ ".

The syntax of terms includes standard $\mathrm{OO}$ primitives for object creation, method call, and field get/set. The let binder in method calls is necessary to describe the provenance of return values. Constructors and methods take predicate parameters that are used statically. The special "field" loc returns the location of an object. The conditional allows equality testing of values.

Concurrent composition ( $\|$ ) is asymmetric. In $N \| M$, the returned value comes from $M$; the term $N$ is available only for side effects. In the sequential composition "let $x=$ $N ; M$ ", $x$ is bound with scope $M$. We elide the let, writing simply " $N$; $M$ " when $x$ does not occur in $M$. We also use standard syntactic sugar in place of explicit sequencing. For example, we may write " $y . f . g$ " to abbreviate "let $x=y . f ; x . g$ ".

Heap elements $(p: c\{\cdots\})$, name restriction $((v p))$ and frames $(a[M])$ are meant only to occur at runtime. The first two of these model the heap, whereas the last models the (potentially distributed) "call stack". We expect that these constructs do not occur in user code. An object name binder $(v)$ is separate from the associated denotation ( $p: c\{\vec{f}=\vec{V}\}$ ), allowing arbitrary graphs of heap objects. (The preceding example indicates that $p$ is located at $a$, with actual class $c$ and fields $\vec{f}=\vec{V}$.) The frame $a[M]$ indicates that $M$ is running under the authority of $a$.

Structural Congruence. Evaluation is defined using a structural congruence on terms. Let $\equiv$ be the least congruence on terms that satisfies the following axioms. The rules in the left column are from [38]. They capture properties of concurrent composition,

\footnotetext{
${ }^{1}$ When writing definitions using classes and methods, we often elide irrelevant bits of syntax, e.g., we leave out the parameters to classes when empty, such as writing Object rather than Object< $<>$. We identify syntax up to renaming of bound names, and write $M[x:=V]$ for substitution of $V$ for $x$ in $M$ (and similarly for other categories). We sometimes write extends for $\triangleleft$ for clarity. We often elide type information. We write " $S \ell(\vec{T} \vec{x})$;" as shorthand for "S $\ell(\vec{T} \vec{x})\{\} "$.
} 
including semi-associativity and the interaction with let. The rules in the right column, inspired by [41], capture properties of distribution. The first of these states that the interpretation of a value is independent of the location at which it occurs. The second states that computation of a frame does not depend upon the location from which the frame was invoked.

Structural Congruence. $\left(M \equiv M^{\prime}\right) \quad($ where $p \notin f n(M))$

\begin{tabular}{ll}
\hline$(M \| N)\|L \equiv M\|(N \| L)$ & $a[V] \equiv V$ \\
$(M \| N)\|L \equiv(N \| M)\| L$ & $a[b[M]] \equiv b[M]$ \\
$((v p) N) \| M \equiv(v p)(N \| M)$ & $a[N \| M] \equiv a[N] \| a[M]$ \\
$M \|((v p) N) \equiv(v p)(M \| N)$ & $a[(v p) N] \equiv(v p) a[N]$ \\
let $x=(L \| N) ; M \equiv L \|(\operatorname{let} x=N ; M)$ & $a[\operatorname{let} x=N ; M] \equiv \operatorname{let} x=a[N] ; a[M]$ \\
let $x=((v p) N) ; M \equiv(v p)(\operatorname{let} x=N ; M)$ & \\
\hline
\end{tabular}

One may view interesting terms as configurations, which we now define. A store $\Sigma$ is a collection of distributed heap terms, $b_{1}\left[p_{1}: c_{1}\{\cdots\}\right]\|\cdots\| b_{m}\left[p_{m}: c_{m}\{\cdots\}\right]$, where each $p_{j}$ is unique. A thread is either a value or a term $a[M]$ that does not contain occurrences of a name restriction or heap term. (A value represents a terminated thread.) An initial thread is a term $a[M]$ such that $M$ additionally contains no blocks. A configuration is a term of the form $(v \vec{p})\left(\Sigma\left\|M_{1}\right\| \cdots \| M_{n}\right)$, where each $M_{i}$ is a thread. A configuration is initial if each of its threads is initial. Evaluation preserves the shape of a configuration up to structural equivalence: If $M$ is a configuration and $M \rightarrow M^{\prime}$ then $M^{\prime}$ is structurally equivalent to a configuration.

Evaluation. The evaluation relation is defined with respect to an arbitrary fixed class table. The class table is referenced indirectly in the semantics through the lookup functions fields and body; we elide the standard definitions.

Evaluation is defined using the following axioms; we elide the standard inductive rules that lift structural equivalence to evaluation $\left(M \rightarrow M^{\prime}\right.$ if $\left.M \equiv N \rightarrow N^{\prime} \equiv M^{\prime}\right)$ and that describe computation in context (for example, $b[M] \rightarrow b\left[M^{\prime}\right]$ if $M \rightarrow M^{\prime}$ ). We discuss the novelties below.

Term Evaluation. $\left(M \rightarrow M^{\prime}\right)$




The rule for new creates an object and returns a reference to it; in the Gordon/Hankin formalism, the heap stays on the left, whereas the return value goes on the right. $p$.loc returns the location of $p$.

Method invocation happens at the callee site, and thus a new frame is introduced in the consequent $b\left[M^{\prime}\right]$. The provenance of the actual parameters is recorded in $\operatorname{Prov}(\vec{W}$, $a, b)$, which is shorthand for $\operatorname{Prov}\left(W_{1}, a, b\right), \ldots, \operatorname{Prov}\left(W_{n}, a, b\right)$. In $M^{\prime}$, the special variable caller is bound to calling principal; there are also standard substitutions for this and the formal parameters. In $L^{\prime}$, the provenance of the return value is recorded in $\operatorname{Prov}(y, b, a)$.

Effects. Effects play a crucial role in the statics, but are ignored by evaluation. In summary, trustworthy processes are required to justify object creation by validating the expectations associated with classes in terms of accumulated effects. Opponent processes, on the other hand, may ignore expectations but are otherwise well typed. We say that a term is safe if the expectations associated with object creation by trusted principals during evaluation are always justified by the accumulated effects. We establish the standard properties of Preservation and Progress. As a corollary, we deduce that well-typed trustworthy processes remain safe when composed with arbitrary opponents.

Our proof of type-safety identifies the key properties required of the logic of effects. Thus, the logic of effects has to support structural rules on the left, support transitivity via cut, and ensure closure of the equality predicate under substitution and reduction. In addition, typechecking of examples (such as the ones that follow) also requires closure of inference under the inference rules of affirmation in the authorization logic of [10], e.g., functoriality of says, distribution of says over conjunction, and $(\alpha \Rightarrow A$ says $\beta) \Rightarrow$ ( $A$ says $\alpha \Rightarrow A$ says $\beta$ ). In this extended abstract, we illustrate the type system using examples; full details can be found in the companion technical report.

\section{Examples}

In these examples, effects are described in a variant of Datalog extended to work over authorization logic. As with regular Datalog, a program is built from a set of Horn clauses without function symbols. In contrast to regular Datalog, the literals can also be in the form of quotes of principals. The well-formed user predicates are typed, with fixed arity. They are always instantiated with pure terms in a type-respecting fashion; pure terms are guaranteed to converge to a value without mutating the heap.

\subsection{Workflow}

In this stateful workflow pattern, a user submits data of type $T$ by creating an object of class SubmittedCell. (For simplicity, we do not address generic types here.) The manager must subsequently approve the data by creating an object of class ApprovedCell. 


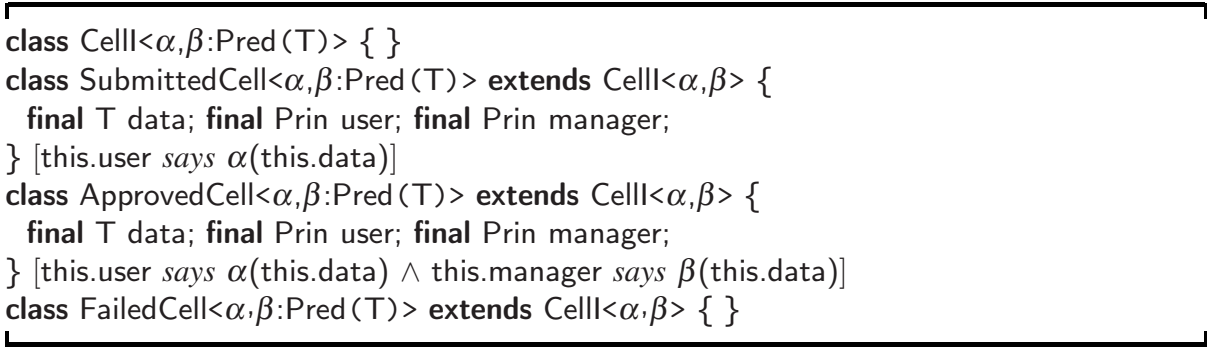

In Cell $\langle\alpha, \beta\rangle, \alpha$ is the predicate that the user establishes on the data in the submission. $\beta$ is the predicate that the manager establishes on the data. The final effect on approved cells represents both approvals in the static types.

The submission and approval objects are generated by a CellFactory in response to receipt of a request object (of class CellReq $\langle\gamma\rangle$ ). The submit method of CellFactory $<\alpha, \beta>$ receives the effect req.loc says $\alpha$ (req.data) on its req parameter. The resulting instance of SubmittedCell $<\alpha, \beta>$ carries this assumption, along with the name of a manager that must approve the request.

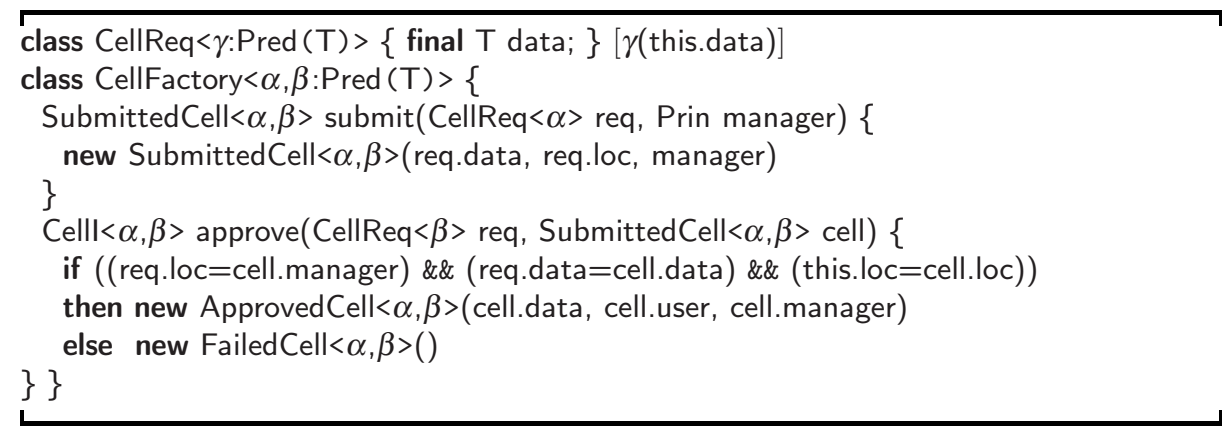

The approve method receives the effect req.loc says $\beta$ (req.data). After checking that req.loc is the same as cell.manager, it may conclude that cell.manager says $\beta$ (req.data). To establish the final effect on the ApprovedCell, the factory must establish that the data in the approval request is the same as the data in the initial request. Further, it must be the case that submit and approve are called upon factories located at the same principal, since the ApprovedCell vouches for both $\alpha$ and $\beta$, although these are validated at different times. If any of the equality tests are missing, the code fails to typecheck.

Visitors for typecases. The class Celll is an interface for cells. The visitor design pattern [42] provides a type-safe way to write code that is dependent on the actual dynamic type/subclass. Thus, we add methods such as visitApprovedCell to class CellV $\langle\alpha, \beta\rangle$ (in general, one such visit method for each subclass). To dispatch to the visitor, the Celll interface is augmented with an accept method, implemented in each subclass; e.g., if $S$ is the return type of the visitor, the implementation of ApprovedCell $<\alpha, \beta>$.accept is:

$$
\text { S accept }(\text { CellV }<\alpha, \beta>\text { v) }\{\text { v.visitApprovedCell(this) }\}
$$

Encoding Provenance. The submission and approval requests described above for the workflow cell do not track provenance. To accommodate provenance tracking, e.g., for the account balance requests discussed in Section 1, we develop an idiom for decorating 
such requests as they are passed from principal to principal. The decorations indicate the provenance of the transmitted data. As usual with a decorator design pattern [42], the $\operatorname{Req}\langle\alpha\rangle$ class is split into three classes: the interface $\operatorname{Req} \mid\langle\alpha\rangle$, the concrete class $\operatorname{Req} C\langle\alpha\rangle$ (which corresponds to the original $\operatorname{Req}\langle\alpha\rangle$ ), and the decorator $\operatorname{Req} \mathrm{D}\langle\alpha\rangle$. We use a visitor to inspect the resulting object. Again, let $\mathrm{T}$ be the type of the request data and $\mathrm{S}$ be the arbitrary return type of the visitor.



Significantly, it is the concrete class $\operatorname{Req} C\langle\alpha\rangle$ that retains the original effect $\alpha$ (this). The decorator, instead, carries an effect concerning the provenance of the decorated data. The effect Prov, used here at type Pred (Req $\mid\langle\alpha\rangle$, Prin, Prin), is a claim about the provenance of one hop of a request. It indicates that this.payload was received from this.src by this.tgt. Thus, the object creation new $\operatorname{ReqD}(p, A, B)$ typechecks only when the static semantics can deduce that $p$ has been received by $B$ from $A$.

To illustrate request decoration, consider the following trustworthy forwardet 2 :

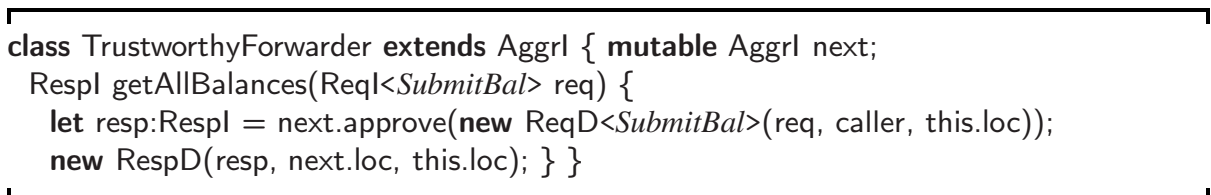

The method body is typechecked in the context of the assertion Prov(req, caller, this.loc), thus permitting the construction of the ReqD object. Similarly, the Prov(resp, next.loc, this.loc) assertion established by the method invocation on next enables the typechecking of the construction of the new RespD object. In contrast, an untrustworthy forwarder might produce an inaccurate provenance decoration for the request, e.g., using new ReqD $<$ SubmitBal $>$ (req, FAKESRC, FAKETGT)). In the following account aggregation example, the principals trusted to provide accurate provenance decorations are specified via the $\theta_{2}$ component of the global policy.

\subsection{Account Aggregation}

Recall, from Figure 1, a rough outline of the protocol: (1) OWNER informs ACCT that AGGR may aggregate its balances (using Acct.addAggr); (2) OWNER requests a summary of its balances from AGGR (using Aggr.getAllBalances); (3) AGGR requests the

\footnotetext{
${ }^{2}$ For reasons of space we omit definition of Aggrl, an interface class with a single getAllBalances method, and classes Respl, RespC, RespD for responses by analogy with non-generic versions of request classes Reql, ReqC, ReqD.
} 
balance from ACCT using Acct.getBalance. Steps (1) and (3) involve communication between the public-facing ACCT and the private MONITOR. In addition, let the principal FORWARDER be trusted to relay messages using the decorator previously discussed. For simplicity, we use a single forwarder and account as well as a single class to represent the code running at each principal. (We follow the convention that field owner references an instance of class Owner located at principal OWNER.) Due to space limitations, we elide the code implementing step (1) of the protocol. We recall that Step (2) of the protocol is initiated by the OWNER, with a call to Aggr.getAllBalances.

The global security policy. The global system policy has the form [OWNER says $\left.\left(\theta_{0}\right)\right] \wedge$ [AGGR says $\left.\left(\theta_{1} \wedge \theta_{2} \wedge \theta_{3}\right)\right] \wedge\left[\right.$ MONITOR says $\left.\left(\theta_{4} \wedge \theta_{5}\right)\right] \wedge\left[\right.$ ACCT says $\left.\theta_{6}\right]$. The predicates $\theta_{0} \ldots \theta_{6}$ are formalized shortly. Informally, $\theta_{0}$ will ensure that the OWNER is authorized to submit balance requests. $\theta_{1}$ and $\theta_{2}$ will characterize the paths that are considered secure. $\theta_{3}$ will ensure that the aggregator only creates requests that arrive from owner on secure paths. $\theta_{4}$ and $\theta_{5}$ will ensure that the MONITOR only accepts requests from owner or from aggregators certified by the owner. $\theta_{6}$ will ensure that the account delegates authorization decisions to the monitor.

The design of the entire program that follows is driven by this global policy, i.e., our code is set up to satisfy the expectations of each principal. Our presentation of the formal policies piecemeal along with the associated classes is only for concise exposition.

Notation. To encode the policy, we use several predicate constructors, which we write in italics. SubmitAggr, with type Pred(Prin), indicates that an aggregator has been submitted for approval. Likewise ApproveAggr indicates that the request was approved. SubmitBal, with type Pred (ReqC $<$ SubmitBal $>$ ), is a claim that a balance request has been submitted. ApproveBal, with type Pred (Reql $\langle$ SubmitBal $\rangle$ ), is a claim that a balance request (perhaps with decorators) has been approved. As described previously, Prov, used here at type Pred (Req $\mid<$ SubmitBal $>$, Prin, Prin), is a claim about the provenance of one hop of a request. CheckedProv, with type Pred (Reql $\langle$ SubmitBal $\rangle$ ), indicates that the provenance of a request has been checked, and is specified using reachability via Prov, incorporating trust in principals that report about each hop.

We assume that the field Monitor.cell is set appropriately. For simplicity, we have hard-coded AGGR and other principals throughout the example code; one may instead use a final field to store principals of interest, deferring the choice to instantiation-time.

Owner. We use some abbreviations and elide the code to check the response received back from the aggregator, which is similar to the visitor used by the aggregator, shown later below. Acct.addAggr expects arguments of type CellReq $<$ SubmitAggr $>$, and Aggr. getAllBalances expects arguments of type Req $\mid\langle$ SubmitBal $\rangle$.

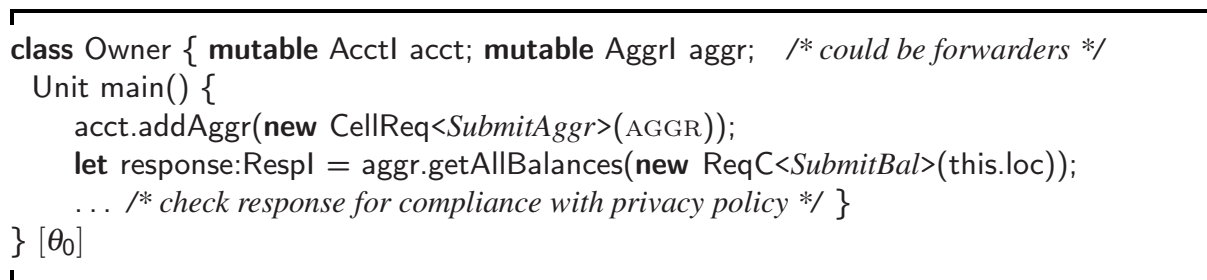


where $\theta_{0}=($ SubmitAggr $($ AGGR $)) \wedge(\operatorname{SubmitBal}(\mathbb{X}):-\mathbb{X}$.data $=\mathbb{X}$. loc $=$ this.loc $)$. This effect indicates that the instantiator must be able to submit the aggregator request and that the instantiator must be able to submit any balance request that it creates, so long as the data field truthfully records its identity. The second requirement is expressed using a Datalog variable $\mathbb{X}$, ranging over values of type $\mathrm{Req} C<$ SubmitBal $>$.

Aggregator. The code uses the following effects.

$\theta_{1}=$ CheckedProv $(\mathbb{X}):-\operatorname{Prov}(\mathbb{X}, \mathbb{S}$, this.loc $), \mathbb{S}=$ OWNER OR $\mathbb{S}=$ FORWARdeR

$\theta_{2}=$ CheckedProv (X.payload) :- FORWARDER says Prov(X.payload, $\mathbb{S}$, FORWARDER), CheckedProv $(\mathbb{X})$

$\theta_{3}=\operatorname{SubmitBal}(\mathbb{X}):-$ OWNER says SubmitBal $(\mathbb{Y}), \mathbb{Y}$. data $=\mathbb{X}$.data $=$ owner, CheckedProv $(\mathbb{Y})$

The first two of these deal with provenance. The base case $\theta_{1}$ validates an object delivered to aggregator from forwarder or owner. $\theta_{2}$ recurses down one level of the decorated object, making explicit the trust on trusted forwarders. Together $\theta_{1}$ and $\theta_{2}$ ensure that a request is deemed valid if it has passed through trusted intermediaries. $\theta_{3}$ allows the aggregator to create new balance requests, if it has checked the provenance of the request: both the new request $\mathbb{X}$ and the old one $\mathbb{Y}$ must have the data field set to OWNER; further, the OWNER must avow that they created the old request.

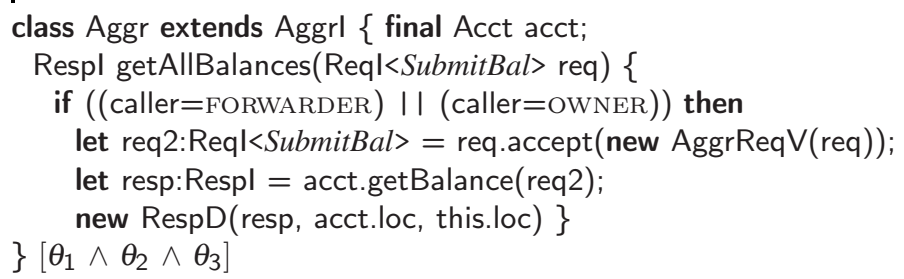

The validation of the creation of req2 uses $\theta_{1}$ to satisfy the effect of the the class AggrReq $V$. The auxiliary class AggrReq $V$ is a visitor to typecase on the request being either a concrete request, or being a forwarded request.

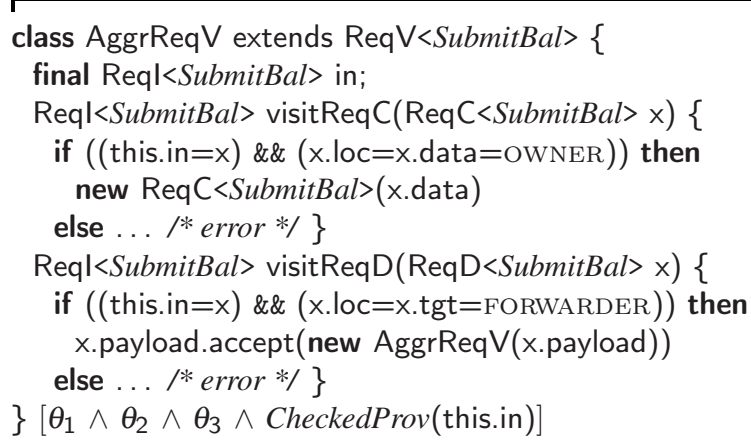

As the visitor traverses the decorators, it maintains the invariant that CheckedProv is true of the object being visited. The visitor updates the effect each time it moves to a new 
element by creating (and using) a new visitor. On callback to visitReq $C$ or visitReqD, the Reql should be the same as the one with the effect; the type system ensures that this is explicitly checked. To type visitReqC requires $\theta_{3}$, which allows us to create the new ReqC located at AGGR. To type visitReqD, we first deduce CheckedProv $(\mathrm{x})$ from this.in $=\mathrm{x}$ and the class effect. Since $x$ is a ReqD, we have $x . l o c$ says Prov (x.payload, x.src, x.tgt). Since $\mathrm{x} \cdot \mathrm{loc}=\mathrm{x} \cdot \operatorname{tgt}=$ FORWARDER and CheckedProv $(\mathrm{x})$, then $\theta_{2}$ yields CheckedProv $(\mathrm{x}$. payload), allowing creation of the new AggrReqV.

The enforcement of the privacy policy of the introduction by the OWNER can be achieved using similar techniques.

Account. Calls to Acct.getBalance are delegated to Monitor.checkBalance, which results in a call back to either Acct.granted or Acct.denied.



Here $\theta_{6}=$ ApproveBal $(\mathbb{X}):-$ MONITOR says ApproveBal $(\mathbb{X})$. Thus, if the granted method is called back, then it must be the case that the monitor approved the request.

Monitor. The effects of the monitor code are expressed using the following predicates.

$\theta_{4}=$ ApproveBal $(\mathbb{X})$ :- OWNER says SubmitBal $(\mathbb{X}), \mathbb{X}$.data=owner

$\theta_{5}=$ ApproveBal $(\mathbb{X}):-$ OWNER says SubmitAggr $(\mathbb{Y})$, this.loc says ApproveAggr $(\mathbb{Y})$,

$\mathbb{Y}$ says SubmitBal $(\mathbb{X}), \mathbb{X} \cdot$ data $=$ ownER

class Monitor \{ mutable Celll<SubmitAggr, ApproveAggr $>$ cell;

Unit checkBalance(Reql $<$ SubmitBal $>$ req, Acct acct) \{

if (req.loc $=$ req. data $=$ OWNER)

then /* audit the request */; acct.granted(new ReqC $<$ ApproveBal $>$ (req.data))

else this.cell.accept(new MonitorCellV(req, acct)) \}

\}$\left[\theta_{4} \wedge \theta_{5}\right]$

class MonitorCellV extends CellV<SubmitAggr, ApproveAggr $>\{$

final Reql<SubmitBal> req; final Acct acct;

Unit visitFailedCell(FailedCell<SubmitAggr, ApproveAggr $>\mathrm{x})\{$ this.acct.denied ()$\}$

Unit visitSubmittedCell(SubmittedCell<SubmitAggr, ApproveAggr $>$ x) $\{$ this.acct.denied() $\}$

Unit visitApprovedCell(ApprovedCell $<$ SubmitAggr, ApproveAggr $>$ x) \{

if $((\mathrm{x}$. loc $=$ this.loc) \&\& (OWNER=x.user) \&\& (this.loc $=x \cdot$ manager)

\&\& (this.req.loc $=x$.data) \&\& (this.req. data $=$ OWNER)

then /* audit the request */; this.acct.granted(new ReqC $<$ ApproveBal $>$ (this.req.data))

\}$\left[\theta_{5}\right]$

else this.acct.denied() $\}$ 
In checkBalance, $\theta_{4}$ establishes the safety of creating the ReqC, whereas $\theta_{5}$ establishes the safety of creating the MonitorCellV.

\section{Conclusion}

TAPIDO is designed to counter the claim that "an application can be mashup-friendly or it can be secure, but it cannot be both." Our model of dynamics adds only two nonstandard features, namely (a) the ability to detect the creator location, and (b) integrity of remote method invocation. We have shown that this suffices to code useful tracking of the provenance of an object reference. Our type system adds (polymorphic) object level effects to standard types. From a programming point of view, this style allows trust-based decisions that are validated by the policy context of the application.

\section{References}

1. Chess, B., O’Neil, Y.T., West, J.: Javascript hijacking. Technical report, Fortify Software (2007), http://www.fortifysoftware.com/news-events/releases/2007/2007-04-02.jsp

2. Lampson, B., Abadi, M., Burrows, M., Wobber, E.: Authentication in distributed systems: Theory and practice. ACM Trans. Comput. Syst. 10(4), 265-310 (1992)

3. Wobber, E., Abadi, M., Burrows, M., Lampson, B.: Authentication in the Taos operating system. ACM Trans. Comput. Syst. 12(1), 3-32 (1994)

4. Abadi, M., Fournet, C., Gonthier, G.: Authentication primitives and their compilation. In: POPL, pp. 302-315 (2000)

5. Landau, S.: Liberty ID-WSF security and privacy overview (2006), http://www.projectliberty.org/

6. Li, N., Mitchell, J.C., Tong, D.: Securing Java RMI-based distributed applications. In: ACSAC, pp. 262-271. IEEE Computer Society, Los Alamitos (2004)

7. Scheifler, B., Venners, B.: A conversation with Bob Scheifler, part I, by Bill Venners (2002), http://www.artima.com/intv/jinisecu.html

8. Gordon, A.D., Pucella, R.: Validating a web service security abstraction by typing. Formal Asp. Comput. 17(3), 277-318 (2005)

9. Abadi, M., Burrows, M., Lampson, B.W., Plotkin, G.D.: A calculus for access control in distributed systems. ACM Trans. Program. Lang. Syst. 15(4), 706-734 (1993)

10. Abadi, M.: Access control in a core calculus of dependency. In: ICFP, pp. 263-273. ACM, New York (2006)

11. Garg, D., Pfenning, F.: Non-interference in constructive authorization logic. CSFW 19, 283 296 (2006)

12. Garg, D., Bauer, L., Bowers, K.D., Pfenning, F., Reiter, M.K.: A linear logic of authorization and knowledge. In: Gollmann, D., Meier, J., Sabelfeld, A. (eds.) ESORICS 2006. LNCS, vol. 4189, pp. 297-312. Springer, Heidelberg (2006)

13. Woo, T.Y.C., Lam, S.S.: A semantic model for authentication protocols. In: IEEE Symposium on Research in Security and Privacy (1993)

14. Fournet, C., Gordon, A.D., Maffeis, S.: A type discipline for authorization in distributed systems. In: CSF, IEEE Computer Society Press, Los Alamitos (2007)

15. Cirillo, A., Jagadeesan, R., Pitcher, C., Riely, J.: Do As I SaY! programmatic access control with explicit identities. In: CSF, IEEE, Los Alamitos (2007) 
16. Gordon, A.D., Jeffrey, A.: Authenticity by typing for security protocols. Journal of Computer Security 11(4), 451-520 (2003)

17. Buneman, P., Tan, W.C.: Provenance in databases. In: SIGMOD Conference, pp. 1171-1173. ACM, New York (2007)

18. Wallach, D.S., Appel, A.W., Felten, E.W.: SAFKASI: a security mechanism for languagebased systems. ACM Trans. Softw. Eng. Methodol. 9(4), 341-378 (2000)

19. Abadi, M., Fournet, C.: Access control based on execution history. In: Proc. Network and Distributed System Security Symp. (2003)

20. Gifford, D.K., Lucassen, J.M.: Integrating functional and imperative programming. In: LISP and Functional Programming, pp. 28-38 (1986)

21. Lucassen, J.M., Gifford, D.K.: Polymorphic effect systems. In: POPL, pp. 47-57 (1988)

22. Talpin, J., Jouvelot, P.: Polymorphic type, region and effect inference. J. Funct. Program. 2(3), 245-271 (1992)

23. Talpin, J., Jouvelot, P.: The type and effect discipline. Inf. Comput. 111(2), 245-296 (1994)

24. Bierman, G., Parkinson, M., Pitts, A.: MJ: An imperative core calculus for Java and Java with effects. Technical Report 563, Cambridge University Computer Laboratory (2003)

25. Greenhouse, A., Boyland, J.: An object-oriented effects system. In: Guerraoui, R. (ed.) ECOOP 1999. LNCS, vol. 1628, pp. 205-229. Springer, Heidelberg (1999)

26. Grothoff, C., Palsberg, J., Vitek, J.: Encapsulating objects with confined types. In: TOPLAS (to appear, 2007)

27. Potanin, A., Noble, J., Clarke, D., Biddle, R.: Featherweight generic confinement. J. Funct. Program. 16(6), 793-811 (2006)

28. Damiani, F., Drossopoulou, S., Giannini, P.: Refined effects for unanticipated object reclassification: Fickle $_{3}$. In: Blundo, C., Laneve, C. (eds.) ICTCS 2003. LNCS, vol. 2841, pp. 97-110. Springer, Heidelberg (2003)

29. DeLine, R., Fähndrich, M.: Enforcing high-level protocols in low-level software. In: PLDI, pp. 59-69 (2001)

30. Dezani-Ciancaglini, M., Yoshida, N., Ahern, A., Drossopoulou, S.: A distributed objectoriented language with session types. In: De Nicola, R., Sangiorgi, D. (eds.) TGC 2005. LNCS, vol. 3705, pp. 299-318. Springer, Heidelberg (2005)

31. Fournet, C., Gordon, A.D., Maffeis, S.: A type discipline for authorization policies. In: Sagiv, M. (ed.) ESOP 2005. LNCS, vol. 3444, pp. 141-156. Springer, Heidelberg (2005)

32. Sabelfeld, A., Myers, A.C.: Language-based information-flow security. IEEE J. Selected Areas in Communications 21(1), 5-19 (2003)

33. Flatt, M., Krishnamurthi, S., Felleisen, M.: Classes and mixins. In: POPL, pp. 171-183 (1998)

34. Igarashi, A., Pierce, B., Wadler, P.: Featherweight Java: A minimal core calculus for Java and GJ. In: OOPSLA (1999)

35. Drossopoulou, S., Eisenbach, S., Khurshid, S.: Is the Java type system sound? Theory and Practice of Object Systems 5(11), 3-24 (1999)

36. Bracha, G., Odersky, M., Stoutamire, D., Wadler, P.: Making the future safe for the past: Adding genericity to the Java programming language. In: OOPSLA, pp. 183-200 (1998)

37. Igarashi, A., Pierce, B.C.: On inner classes. Information and Computation 177(1), 56-89 (2002)

38. Gordon, A.D., Hankin, P.D.: A concurrent object calculus: Reduction and typing. In: Proceedings HLCL'98, ENTCS (1998)

39. Cardelli, L.: A language with distributed scope. In: POPL, pp. 286-297. ACM Press, New York (1995) 
40. Jeffrey, A.S.A.: A distributed object calculus. In: Proc. Foundations of Object Oriented Languages (2000)

41. Castellani, I.: Process algebras with localities. In: Handbook of Process Algebra, NorthHolland, pp. 945-1045 (2001)

42. Gamma, E., Helm, R., Johnson, R., Vlissides, J.: Design Patterns. Addison-Wesley, Reading (1995) 